\title{
Mechanisms and relevant genes of drug resistance in gastric cancer
}

\author{
Xingwang Kuai, Lizhou Jia \\ Department of Pathology, Nanjing Medical University, Nanjing 211166, China
}

\begin{abstract}
Gastric cancer (GC) is one of the most common malignancies in China, and chemotherapy is an important treatment for GC. However, drug resistance remains the main barrier to successful chemotherapy. Drug resistance is a complex phenomenon resulting from a combination of factors and mechanisms. The number of known relevant genes implicated in this phenomenon is growing rapidly. This review focuses on the mechanisms involved in the occurrence of drug resistance and explores the functions of several relevant genes in GC chemotherapy resistance.
\end{abstract}

Keywords: chemotherapy, drug resistance, gastric cancer

\section{INTRODUCTION}

Gastric cancer (GC) is the fourth most common malignancy in the world and more than $70 \%$ of cases occur in developing countries, especially Eastern Asia $^{[1,2]}$. Due to certain dietary habits including highsalt diets and a preference for pickled foods, as well as eating disorders, GC is the second most common cancer and represents the second leading cause of cancer death in $\mathrm{China}^{[3]}$. The main treatments for gastric cancer include surgery, chemotherapy, radiotherapy, molecular targeted therapy and so on. The 5-year survival rate of patients with early gastric cancer can reach $95 \%$ after surgery. However, gastric cancer is characterized by metastasis and drug resistance, and less than 20 percent of patients with advanced gastric cancer will survive five years ${ }^{[4-5]}$. Most Chinese GC patients are initially diagnosed with late stage GC because of atypical symptoms in the early stages coupled with uncommon gastroscopy. Chemotherapy plays an important role in perioperative and palliative treatment in GC, but chemotherapy failure is usually caused by drug resistance, leading to shorter overall

*Correspondence to: Lizhou Jia, Department of Pathology, Nanjing Medical University, Nanjing 211166, China. E-mail: nmgjlz@163.com

Confiict of interests: The authors have declared no conflict of interests. survival and a poorer quality of life.

GC cell resistance to chemotherapy drugs is divided into two categories, namely: primary drug resistance and multidrug resistance. Primary drug resistance is defined as cancer cells that show resistance to a single chemotherapeutic agent without showing any resistance to other kinds of chemotherapeutics. Multidrug resistance (MDR, also known as cross resistance) occurs when patients develop resistance to multiple agents with different structures and/or mechanisms after prolonged exposure to a single anticancer drug. Drug resistance can also be classified as intrinsic drug resistance and acquired drug resistance. Intrinsic drug resistance indicates patients with a pre-existent drug resistance to a particular chemotherapeutic agent, while acquired drug resistance develops after successful chemotherapeutic treatment. This review briefly probes common mechanisms and relevant genes implicated in GC drug resistance, as well as different molecular modulators employed to reverse cancer drug resistance.

\section{MECHANISMS OF GC DRUG RESISTANCE}

\section{Increased drug efflux pumps}

Human ATP-binding cassette (ABC) transporters are transmembrane proteins that deliver multitudinous molecules across lipid bilayers. They can obtain 
energy by hydrolyzation of ATP to carry anticancer drugs, resulting in decreasing intracellular drug concentrations or a varied drug distribution. At least 12 members of the ABC superfamily are connected with drug resistance in laboratory studies, while only three transporters have been confirmed in multiple studies as playing chemoresistant roles in vivo, which include: $\mathrm{P}$-glycoprotein (P-gp/ABCBl/MDR1), multidrug resistance protein 1 (MRPl/ABCCl), and breast cancer resistance protein $(\mathrm{BCRP} / \mathrm{ABCG} 2)^{[6]}$. In addition to $\mathrm{ABC}$ transporters, lung resistance protein (LRP) is also an integral membrane protein related to drug resistance, but does not require ATP. It is reported that drug efflux pumps greatly influence GC cell drug resistance $^{[7-8]}$.(Fig. 1)

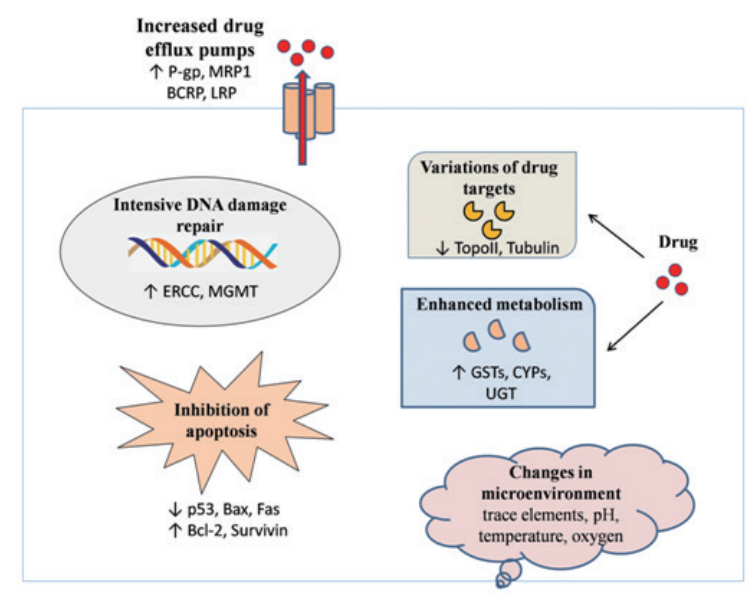

Fig. 1 Mechanisms and relevant genes implicated in GC drug resistance.

\section{Enhanced metabolism to drugs}

Some enzymes can transform external toxins or intermediates into innocuous substances, rendering anticancer drugs inactive to cancer cells. The most common enzymes involved in drug metabolism include glutathione-S-transferases (GSTs), cytochrome P450s (CYPs), and uridine diphosphate glucuronosyltransferase (UGT). CYPs can convert hydrophobic compounds into inactive polar products suitable for excretion via the kidneys ${ }^{[9]}$. UGTs can catalyze the inactivation of cytotoxic drugs through the formation of hydrophilic conjugates ${ }^{[10]}$. Some CYP and GST isoenzymes have been confirmed to be overexpressed in GC tissues, which may promote gastric carcinoma $\mathrm{MDR}^{[11]}$.

\section{Variations of drug targets}

Chemotherapeutic efficacy depends to a large extent on the profusion of target molecules. Target protein mutation or decreased expression often represents declining chemosensitivity in human cancer. Topoisomerase II (Topo II ) is an important cell proliferation regulator and has been confirmed as the target of many structurally diverse antitumor drugs ${ }^{[12]}$, as quantitative and qualitative changes in Topo II have been found to significantly influence cancer cell drug resistance. Tubulin is the target of paclitaxel, which can inhibit spindle formation, and any changes in tubulins will affect tumor tolerance to paclitaxel therapy ${ }^{[13]}$. The expressions of TopoII and tubulin are related to chemosensitivity in gastric cancer ${ }^{[14,15]}$.

\section{Intensive DNA damage repair}

Cancer cells are able to strengthen DNA repair which help the cell withstand the effects of chemotherapeutics (which conversely acts by suppressing tumors by directly or indirectly damaging cellular DNA). Excision repair cross-complementation (ERCC) genes play a vital role in nucleotide excision repair. The ERCCl - XPF nuclease is encoded by ERCCl and ERCC4 which not only nicks on the 5' side of the damaged DNA strand but also repairs DNA interstrand crosslinks ${ }^{[16]}$. $\mathrm{O}^{6}$-methylguanine DNA methyltransferase (MGMT) can repair the damaged DNA by inducing the demethylation of $\mathrm{O}^{6}$-methylguanine, which is caused by alkylating agents ${ }^{[17]}$. Due to this mechanism, DNA repair genes are able to impair chemotherapeutic effectiveness in GC patients ${ }^{[18]}$.

\section{Inhibition of apoptosis}

The therapeutic outcome of chemotherapy largely depends on the reaction of the cells' apoptotic mechanism, namely if the chemotherapeutic drugs have damaged the DNA irrevocably. There are two drug resistance mechanisms induced by apoptotic inhibition: low expression of apoptotic genes and overexpression of antiapoptotic genes. The former mainly involves p53, Bax and Fas, and the latter consists of Bcl-2, survivin and others. P53 is a tumor suppressor gene prone to mutation, which induces cell apoptosis and activates apoptosis-inducing factors like Fas and $\mathrm{Bax}^{[19]}$. Fas is a member of the tumor necrosis factor superfamily, and the low expressed Fas (or the lack of its receptor) can inhibit cell apoptosis ${ }^{[20]}$. Survivin belongs to the apoptotic inhibitory protein family and its role is to interdict apoptotic processes by directly inhibiting caspase 3 and caspase $7^{[21]}$. Apoptosis related genes greatly regulate the drug resistance of GC cells $^{[22-24]}$.

\section{Changes in cell microenvironment}

Trace elements such as calcium, zinc and selenium can affect the sensitivity of cancer cells to chemo- 
therapeutic drugs through a variety of pathways. Furthermore, $\mathrm{pH}$, temperature, oxygen concentration and substrate nutrient conditions also make a difference to drug resistance.

\section{GENES RELATED TO GC DRUG RE- SISTANCE}

\section{P-gp}

$\mathrm{P}$-gp is a $170 \mathrm{kDa}$ phosphorylated transmembrane glycoprotein encoded by MDR1/ABC and MDR3/ $\mathrm{ABCB} 4$, which maps to the long arm of chromosome 7q21. P-gp consists of 1280 amino acids, including two tandem repeat sequences and one joining region. Each tandem repeat sequence is composed of six hydrophobic transmembrane alpha helixes and one hydrophilic nucleotide binding domain(NBD). (containing two ATP binding sites) which can provide energy for transport by hydrolyzing ATP.

P-gp shows prominent expression in excretory tissues and at physiological barriers, including: the gastrointestinal tract, the renal proximal tubule, and the blood-brain barrier. P-gp expression is higher in GC tissues than in neighboring tissues, and increased $\mathrm{P}$-gp protein levels in GC have been associated with increased differentiation and lymph node metastasis $^{[25]}$. P-gp transports a wide range of electroneutral or electropositive hydrophobic compounds including taxol, vincristine, etoposide, and many others that are clinically indispensable in cancer treatment ${ }^{[26-28]}$. The clinical application of P-gp inhibitors has always been an area of hot research, but there are several negative factors: the insufficient affinity and specificity of the inhibitors; compensatory adjustment of optional drug transporters; transporter polymorphisms in patients; and the expression of P-gp in normal tissues.

\section{MRP1}

MRP1 is a $190 \mathrm{kDa}$ transmembrane protein with significant substrate overlap with P-gp. The MRP1 gene is located on chromosome 16p13.1, and the MRPl gene encodes the 1531 amino acid protein. MRP1 is made up of three membrane-spanning domains $\left(\mathrm{MSD}_{0}, \mathrm{MSD}_{1}\right.$ and $\mathrm{MSD}_{2}$ ) and two NBDs. $\mathrm{MSD}_{1}$ and $\mathrm{MSD}_{2}$ which constitute the pore through which substrates are transported.

MRP1 is widely distributed in normal tissues, particularly the testicles, kidney and placenta, and can be detected in subcellular organelles. MRPl expression in GC tissues is higher than that in matched paracarcinoma tissues ${ }^{[29]}$. MRP1 transports many clinical chemotherapeutics: etoposide, irinotecan, methotrexate, doxorubicin, vincristine, and mitoxantrone ${ }^{[30-31]}$. While several P-gp inhibitors tested in adult cancers can also suppress MRP1, few specific inhibitors for MRPl have been evaluated clinically. Cyclohexyllinked tricyclic isoxazoles and flavonoid derivatives show selectivity for MRP1, but more clinical studies need to be done to confirm these results ${ }^{[32-33]}$.

\section{BCRP}

BCRP is a $72 \mathrm{kDa}$ protein consisting of 665 amino acids. Its encoding gene maps to chromosome $4 \mathrm{q} 22$. BCRP monomers have only one NBD and one MSD; therefore, BCRP is a half-transporter. Haider et al. proved that BCRP functions as homodimers in the ${ }^{[34]}$ of membrane.

As a universal marker of stem cells, BCRP is widely found in the stem cells of all major tissues ${ }^{[35]}$. BCRP is also expressed in the intestine, liver, bloodbrain barrier, and other tissues. Expression of BCRP is upregulated in GC compared with adjacent non-neoplastic tissue and is implicated in carcinoma differentiation $^{[36]}$. Substrates of BCRP include: mitoxantrone, camptothecin analogs, aminopterin, and others. Furthermore, BCRP can inhibit the effects of prazosin and glibenclamide $^{[37]}$. Fumitremorgin C, elacridar, tyrosine kinase inhibitors, and many other BCRP inhibitors have been reported, but few small molecule modulators have shown promising effects in clinical trials ${ }^{[38]}$.

\section{LRP}

LRP is a $110 \mathrm{kDa}$ protein originally identified in lung cancer MDR cell lines. The LRP gene is located on chromosome 16pll.2 with high similarity to genes of major vault proteins in slime molds and brown mice. LRP is distributed in the nuclear pore complex(NPC) and cytoplasm ${ }^{[39,40]}$. There are two potential mechanisms of LRP-mediated drug resistance:

(1) LRP prevents anticancer drugs from entering the nucleus through the NPC and pumps out drugs in the nucleus; and (2) LRP takes part in vesicular transport to remove chemotherapeutics.

LRP is mainly involved in secretion in the epithelium. The level of expression of LRP in GC tissues is higher than in noncancerous tissues, however LRP has not been correlated with clinical and/or pathological characteristics of $\mathrm{GC}^{[41]}$. Overexpression of LRP reduces cancer cell sensitivity to doxorubicin, etoposide, vincristine, paclitaxel, and cisplatinum. Cyclosporin A, hydrogen epoxy methylquinone, tyroservatide, as well as many other agents can regulate LRP expression, but reversing LRP-mediated drug resistance clinically will require greater effort ${ }^{[42]}$.

\section{GSTs}

GSTs belong to the phase II detoxification enzyme 
group and consist of seven subclasses $(\alpha, \mu, \pi, \theta, \kappa, \sigma$, and $\zeta$ ). GSTs catalyze the conjugation of glutathione (GSH) with a broad range of electrophilic exogenous substrates. The direct catalytic action on drug metabolites (rather than on the parent drugs) lowers reactivity and increases the water solubility of these drugs ${ }^{[43]}$. In addition, GSTs regulate cell apoptosis by inhibiting the JNK signaling pathway ${ }^{[44]}$.

GSTs are expressed in a wide range of normal tissues, and different tissues express different levels of GST isoenzymes. GST $\pi$ is the most highly expressed GST isoenzyme in cancer tissues ${ }^{[45]}$. The expression level of GST $\pi$ in GC tissues is higher than in normal tissues, and the expression of GST $\pi$ is connected with tumor invasion and lymphatic metastasis ${ }^{[46]}$. Anticarcinogens that can be detoxified by GST $\pi$ include chlorambucil, cis-platinum, mitoxantrone, cyclophosphamide, and others ${ }^{[43]}$. Etacrynic acid is an earlier example of a GST inhibitor. Etacrynic acid can increase the sensitivity of tumors to alkylating agents; however, serious side effects (such as diuresis) hamper the clinical application of this molecule ${ }^{[47]}$. GSH analogues like TER199 (which are competitive inhibitors of both GSH and GSTs) can also induce cancer cell apoptosis through the MAPK pathway, but can also cause bone marrow hyperplasia, limiting the utility of these inhibitors ${ }^{[48]}$. NBDHEX and its analogues are immune to drug pumps, even those that are highly expressed (particularly the analogues MC3165 and MC3181, which show higher specificity and water solubility) have the potential to be used as oral GST $\pi$ inhibitors $^{[49-51]}$.

\section{Topo II}

Mammalian Topo II is divided into Topo II $\alpha$ (170 kDa) and Topo II $\beta$ (180 kDa), encoded on chromosomes 17q21-22 and 3p24, respectively. Topo II forms cleavable complexes with DNA to catalyze DNA untwisting. Anticancer drugs targeting Topo II induce single or double-strand breaks by inhibiting Topo II catalytic activity (Topo II inhibitor) or prolonging the half-life of DNA-Topo II complex (Topo II toxicant).

Topo II $\alpha$ is mainly distributed in proliferating the cell nucleoplasm, and Topo II $\beta$ is widely found in the nucleolus of almost all cells. Expression levels of Topo II in normal gastric mucosal tissues is lower than in GC tumors, and the expression of Topo II is associated with tumor differentiation and lymphatic metas$\operatorname{tasis}^{[52]}$.Topo II is an important target for many tumor chemotherapeutics used in clinic. Topo II inhibitors include aclarubicin, merbarone, ICRF-193 and others.
Topo II toxicants include etoposide, doxorubicin, mitoxantrone, as well as other similar agents ${ }^{[53]}$. With the potential to reverse MDR and reduce side effects, the combination of anticancer drugs that can inhibit both topoI and topo II has become a hot area of research.

\section{Bcl-2 and Bax}

The Bcl-2 protein family plays an important role in regulating apoptosis through the intrinsic mitochondrial apoptotic pathway. Bcl-2 is a common prosurvival protein, whereas Bax is a member of the proapoptotic Bcl-2 family. When Bcl-2 is in the majority, heterodimers consist of $\mathrm{Bcl}-2$ and $\mathrm{Bax}$, preventing Bax from damaging mitochondria. When Bax is dominant, homodimers made up of Bax can damage mitochondrial membrane structures and promote the release of pro-apoptotic factors, leading to cell apoptosis.

The expression level of $\mathrm{Bcl}-2$ in precancerous tissues is significantly lower than that in GC tissues; conversely Bax shows a higher expression level in precancerous tissues ${ }^{[54]}$. In gastric tumors, the expression level of Bcl-2 is inversely connected with the apoptotic index while Bax behaves the opposite way ${ }^{[55]}$. Bcl-2 inhibitors, such as ABT-737and S-055746, have been studied in clinical trials, and ABT-199 (venetoclax) has been approved for the treatment of $17 \mathrm{p}$ deleted CLL patients ${ }^{[56]}$. Several direct Bax activators (including Bim, SAHB, BAM7, and SMBAs) have been identified to effectively induce Bax-mediated apoptosis in vitro and in vivo but their clinical application is still a long way off ${ }^{[57]}$.

\section{CONCLUSION AND FUTURE PERSPEC- TIVES}

Progress has been made in our understanding of the mechanisms involved in GC chemotherapy resistance, and the functions of numerous resistance-related genes have been investigated in the last decades. However, the universal mechanisms of GC chemotherapy resistance are still unclear, and the understanding of complex interactions between drug-resistant molecules will require more researches. The manipulation of resistance-related genes has enormous potential to mitigate GC drug resistance, however most molecular inhibitors targeting drug-resistant proteins show poor therapeutic efficacy due to specificity, pharmacokinetics, as well as other factors. More comprehensive investigations into drug resistance are encouraged, and will undoubtedly contribute to the development of anticancer drugs and the optimization of chemotherapy regimens. Drug resistance in GC will most likely be greatly improved with future research. 


\section{References}

[1] Ferlay J, Soerjomataram I, Dikshit R, et al. Cancer incidence and mortality worldwide: sources, methods and major patterns in GLOBOCAN 2012[J]. Int $J$ Cancer,2015,136(5):E359-E386.

[2] Van CE, Sagaert X, Topal B, et al. Gastric cancer[J].Lancet,2016,388(10060):2654.

[3] Chen W, Zheng R, Baade PD, et al. Cancer statistics in China, 2015[J]. CA Cancer J Clin,2016,66(2):115-132.

[4] Yoon H, Kim N. Diagnosis and management of high risk group for gastric cancer[J]. Gut Liver,2015,9(1):5-17.

[5] Duo-Ji MM, Ci-Ren BS, Long ZW, et al. Short-term efficacy of different chemotherapy regimens in the treatment of advanced gastric cancer: a network metaanalysis[J]. Oncotarget,2017,8(23):37896-37911.

[6] Fletcher JI, Williams RT, Henderson MJ, et al. ABC transporters as mediators of drug resistance and contributors to cancer cell biology[J]. Drug Resist Updat,2016,26:1-9.

[7] Li XQ, Wang L, Lei Y, et al. Reversal of P-gp and BCRP-mediated MDR by tariquidar derivatives[J]. Eur J Med Chem,2015,101:560-572.

[8] Yan J, Dang Y, Liu SY, et al. LncRNA HOTAIR promotes cisplatin resistance in gastric cancer by targeting miR-126 to activate the PI3K/AKT/MRP1 genes[J]. Tumor Biology,2016,37(12):16345-16355.

[9] Manikandan P, Nagini S. Cytochrome P450 structure, function and clinical significance: a review[J]. Curr Drug Targets,2018,19(1):38-54.

[10] Bock KW. The UDP-glycosyltransferase (UGT) superfamily expressed in humans, insects and plants: Animalplant arms-race and co-evolution[J]. Biochem Pharmacol,2016,99:11-17.

[11] Simsek GG, Oguztuzun S, Bozer B, et al. Expressions of CYP and GST isoenzymes in human gastric tumor and non-tumor tissues[J]. Uhod-Uluslararasi HematolojiOnkoloji Dergisi,2018,28(1):36-44.

[12] Pendleton M, Lindsey RH, Felix CA, et al. Topoisomerase II and leukemia[J]. Ann N Y Acad Sci,2014,1310):98-110.

[13] Nepali K, Ojha R, Lee HY, et al. Early investigational tubulin inhibitors as novel cancer therapeutics[J]. Expert Opin Investig Drugs, 2016, 25(8):917-936.

[14] Geng M, Wang L, Chen X, et al. The association between chemosensitivity and Pgp, GST- $\pi$ and Topo II expression in gastric cancer[J]. Diagn Pathol, 2013, 8(1):198.

[15] He W, Zhang D, Jiang J, et al. The relationships between the chemosensitivity of human gastric cancer to paclitaxel and the expressions of class III $\beta$-tubulin, MAPT and survivin[C]. 2014 National Cancer Rehabilitation and Palliative Medicine Conference. 2014.

[16] Manandhar M, Boulware KS, Wood RD. The ERCC1 and ERCC4 (XPF) genes and gene products[J]. Gene,2015,569(2):153-161.

[17] Christmann M, Verbeek B, Roos WP, et al. O(6)-Methylguanine-DNA methyltransferase (MGMT) in normal tissues and tumors: enzyme activity, promoter methyla- tion and immunohistochemistry[J]. Biochim Biophys Acta, 2011, 1816(2):179-190.

[18] Won DY, Kim SH, Hur H, et al.Chemotherapeutic responsibility according to polymorphism of ERCC1, XRCC1 and GSTP1 in gastric cancer patients receiving oxaliplatin based chemotherapy[J].J Korean Surg Soc,2010,78(6):350-356.

[19] Speidel D.Transcription-independent p53 apoptosis: an alternative route to death[J].Trends Cell Biol,2010,20(1):14-24.

[20] Villa-Morales M, Fernández-Piqueras J.Targeting the Fas/FasL signaling pathway in cancer therapy[J].Expert Opin Ther Targets,2012,16(1):85-101.

[21] Chen X, Duan N, Zhang C, et al. Survivin and tumorigenesis: molecular mechanisms and therapeutic strategies[J]. J Cancer,2016,7(3):314-323.

[22] Wang X, Lin Y, Lan F, et al. BAX and CDKN1A polymorphisms correlated with clinical outcomes of gastric cancer patients treated with postoperative chemotherapy[J]. Med Oncol,2014,31(11):249.

[23] Jin J, Xiong Y, Cen B.Bcl-2 and Bcl-xL mediate resistance to receptor tyrosine kinase-targeted therapy in lung and gastric cancer[J]. Anticancer Drugs,2017,28(10):1141-1149.

[24] Han Y, Song C, Wang J, et al. HOXA13 contributes to gastric carcinogenesis through DHRS2 interacting with MDM2 and confers 5-FU resistance by a p53-dependent pathway[J]. Mol Carcinog, 2018, 57(6):722-734.

[25] Hongpei W, Junhai I, Yuan Z, et al. Expression and clinical significance of P-gp,GST- $\pi$,Topo II in gastric carcinom[J]. Modern Oncology,2010,18(9):1788-1791.

[26] Yang CH, Wang C, Ojima I, et al. Taxol analogues exhibit differential effects on photoaffinity labeling of $\beta$-Tubulin and the multidrug resistance associated $\mathrm{P}$ Glycoprotein[J]. J Nat Prod,2018,81(3):600-606.

[27] Cheon JH, Kim JY, Lee BM, et al. P-gp inhibition by XL019, a JAK2 inhibitor, increases apoptosis of vincristine-treated resistant KBV20C cells with increased $\mathrm{p} 21$ and $\mathrm{pH} 2 \mathrm{AX}$ expression[J]. Anticancer Res,2017,37(12):6761-6769.

[28] Fujita-Hamabe W, Nishida M, Nawa A, et al. Etoposide modulates the effects of oral morphine analgesia by targeting the intestinal P-glycoprotein[J]. J Pharm Pharmacol,2012,64(4):496-504.

[29] Xie J, Chen H, Jiang Y, et al. Expression and significance of multidrug resistance-associated protein-1 in gastric carcinoma[J]. Shi Jie Hua Ren Xiao Hua Za Zhi,2007,15(10):1092-1095.

[30] Lu JF, Pokharel D, Bebawy M. MRP1 and its role in anticancer drug resistance[J].Drug Metab Rev,2015,47(4):406419.

[31] Peterson BG, Tan KW, Osa-Andrews B, et al. Highcontent screening of clinically tested anticancer drugs identifies novel inhibitors of human MRP1 (ABCCl)[J]. Pharmacological Research,2017,119:313-326.

[32] Barmade MA, Murumkar PR, Sharma MK, et al. Medicinal chemistry perspective of fused isoxazole derivatives[J]. 
Curr Top Med Chem,2016,16(26):2863-2883.

[33] Obreque J E, Sun Q, Bernhardt G, et al. Flavonoid derivatives as selective $\mathrm{ABCCl}$ modulators: Synthesis and functional characterization [J]. Eur J Med Chem, 2016, 109:124-33.

[34] Haider AJ, Briggs D, Self TJ, et al. Dimerization of ABCG2 analysed by bimolecular fluorescence complementation[J].PLoS One, 2011, 6(10):e25818.

[35] Fatima S, Zhou S, Sorrentino BP. Abcg2 expression marks tissue-specific stem cells in multiple organs in a mouse progeny tracking model[J]. Stem Cells,2012,30(2):210-221.

[36] Wang N, Chen L, Wei B, et al. Expression of ABCG2 in human gastric carcinoma[J]. Chin-Germ J of Clin Oncol,2010,9(3):145-148.

[37] Stacy AE, Jansson PJ, Richardson DR. Molecular pharmacology of ABCG2 and its role in chemoresistance[J]. Mol Pharmacol,2013,84(5):655-669.

[38] Peña-Solórzano D, Stark SA, König B, et al. ABCG2/ BCRP: specific and nonspecific modulators[J].Med Res Rev,2017,37(5):987-1050.

[39] Wood N, Streckfus CF. The expression of lung resistance protein in saliva: a novel prognostic indicator protein for carcinoma of the breast[J]. Cancer Invest,2015,33(10):510-515.

[40] Zuo Y, Yang D, Yu Y, et al. Niclosamide enhances the cytotoxic effect of cisplatin in cisplatin-resistant human lung cancer cells via suppression of lung resistance-related protein and c-myc[J]. Mol Med Rep, 2018, 17(3):3497-3502.

[41] Wang J, Li X, Zhang K, et al. Expressions and significance of LRP,GST- $\pi$ and GCS in gastric cancer tissues[J]. Chin J Cancer Prev Treat, 2011, 18(11):859-862.

[42] Emonard H, Théret L, Bennasroune AH, et al. Regulation of LRP-1 expression: make the point[J]. Pathol Biol (Paris),2014,62(2):84-90.

[43] Sau A, Pellizzari Tregno F, Valentino F, et al. Glutathione transferases and development of new principles to overcome drug resistance[J]. Arch Biochem Biophys,2010,500(2):116-122.

[44] Marchewka Z, Piwowar A, Ruzik S, et al. Glutathione $\mathrm{S}$-transferases class $\mathrm{Pi}$ and $\mathrm{Mi}$ and their significance in oncology[J]. Postepy Hig Med Dosw (Online), 2017, 71(0):541-550.

[45] Allocati N, Masulli M, Di Ilio C, et al. Glutathione transferases: substrates, inihibitors and pro-drugs in cancer and neurodegenerative diseases[J]. Oncogenesis, 2018,7(1):8.

[46] Liu L, Jiang Y, Li L, et al.Expressions and significance of HER2,COX-2 and GST- $\pi$ in human gastric carcinoma[J]. J Modern Oncol,2013,21(5):1066-1069.

[47] Groom H, Lee M, Patil P, et al.Inhibition of human glutathione transferases by dinitronaphthalene derivatives $[\mathrm{J}]$. Arch Biochem Biophys, 2014, 555-556(8):71-76.

[48] Raza A, Galili N, Smith S, et al.Phase 1 multicenter dose-escalation study of ezatiostat hydrochloride (TLK199 tablets), a novel glutathione analog prodrug, in patients with myelodysplastic syndrome[J].Blood, 2009, 113(26):6533-6540.

[49] De Luca A, Rotili D, Carpanese D, et al. A novel orally active water-soluble inhibitor of human glutathione transferase exerts a potent and selective antitumor activity against human melanoma xenografts[J].Oncotarget, 2015, 6(6):4126-4143.

[50] Rotili D, De Luca A, Tarantino D, et al. Synthesis and structure--activity relationship of new cytotoxic agents targeting human glutathione-S-transferases[J]. Eur J Med Chem,2015,89:156-171.

[51] Luisi G, Mollica A, Carradori S, et al. Nitrobenzoxadiazolebased GSTP1-1 inhibitors containing the full peptidyl moiety of (pseudo)glutathione[J]. J Enzyme Inhib Med Chem, 2016, 31(6):924-930.

[52] Junmei L, Li SF, Li Y, et al. Expression of Topo-II,GST- $\pi$ in gastric cancer tissues and their significance[J]. Cancer Res Clin,2014,26(7):446-450.

[53] Lee KC, Bramley RL, Cowell IG, et al. Proteasomal inhibition potentiates drugs targeting DNA topoisomerase II[J]. Biochem Pharmacol,2016,103)29-39.

[54] Chen Z, Lu L, Feng J, et al. Expression of Bcl-2 and Bax in precancerous and gastric cancer tissues[J]. Pract Clin Med,2015,16(8):13-14.

[55] Wu D, Li C. Relationship between Bcl-2 and Bax expressions in gastric carcinoma and precancerous lesions and apoptosis[J]. J Zunyi Med Univ,2014,37(2):201-203.

[56] Vervloessem T, Kerkhofs M, La Rovere RM, et al. Bcl-2 inhibitors as anti-cancer therapeutics: The impact of and on Calcium signaling[J]. Cell Calcium,2018,70:102-116.

[57] Liu Z, Ding Y, Ye N, et al. Direct activation of Bax protein for cancer therapy[J]. Med Res Rev,2016,36(2):313-341.

(Received 18 May 2018, Revised 10 June 2018, Accepted 15 June 2018) 